\title{
The Research on Key Technologies and Working Process of Modern Industrial Robot Vision
}

\author{
Chuansong Zhou ${ }^{1, \mathrm{a} *}$ and Zhengyi $\mathrm{Hu}^{1}$ \\ ${ }^{1}$ Changchun automobile industry institute, Changchun, 130000, China. \\ *The Corresponding author: Izyl068052@126.com
}

Keywords: Industrial robot; Vision-guided; Dynamic grasping; Image segmentation

\begin{abstract}
As the way of non-contact mode of sensing, the application of industrial robots in production line is more easy to adapt to the change of product and put work piece. Robot vision provides the real-time target state information of the object, so it has been considered to be one of the most important awareness. Vision-guided system can not only know robots crawl or tracking products, can also locate random parts. At the same time, the inspection and measure can able to form a database. In this article, the development and working process of the modern industrial robot vision technology are researched and analyzed. The composition of robot vision-guided system is introduced and explored in detail. Some main methods and technologies have also been explained.
\end{abstract}

\section{Introduction}

Industrial robot began in the 1960s, it mainly serves for the industrial product. It have been widely used in chemical production, equipment installation, road construction, machinery manufacturing, mining and other fields. Industrial robot has become the irreparable important equipment in the advanced manufacturing industry for its advantages, such as save labor, reduce costs and raising labour productivity.

At present, international industrial robot manufacturers are mainly distributed in Europe and Japan, the main brands include ABB, KUKA, FANUC and COMAU. Industrial robot has changed the mode of production of enterprise, improve the market competitiveness and become the core factor of enterprise transformation. It is used primarily in the automobile manufacturing, in the 70s, research emphasis of robot is the use of external sensors to enhance the level of the intelligent industrial robot $[1,2,3]$. However, in recent years, the advantages of robots has been increasingly significant, the use of industry is becoming more and more diversified, such as military, aerospace, biological, medical, agriculture, forestry and even services.

Robot technology research in China began in the late 70s to 90s. China has invested more than 20 robot base of scientific research and industrialization base. At present, the research in industrial robot has made some breakthrough results, such as robot vision of tsinghua university research team has developed a set of CMMS multi-sensor vision system based on CAD model.

\section{The Robot Vision-Guided System.}

The whole car production process cycle basically adopts the technology of the robot visual guide. Robot visual guide technology can be applied to the grasp of conveyor belt ,such as vehicle door,side surround parts and roof.This can greatly reduce the artificial operation cost and the complexity of equipment to improve the degree of system flexible[4,5].In many industries, for example welding tracking, packing and palletizing are using visual guide technology. With the progress of science and technology, national enterprise pay more and more attention to investment in industrial robot.In addition, the constant development and mature vision-guided system and costs continue to reduce,the application of visual-guided system will be more widely in the future.

Although the visual guide system on different workplace the hardware configuration will be different, but it is pretty much the same. When the system each of function module began to run, with the target workpiece trigger signal,the visual system began to run.After the vision system through the camera to the target image acquisition,computer system began to preliminary judgment 
for these coordinate point. If the feature points of data within the scope of the expectations, the visual system will be according to the internal algorithm to work out the image coordinates conversion into the variation of workpiece coordinate.However, for the most part,there are many scattered on the workpieces stage.The workflow of vision-guided system is roughly as shown in Fig. 1.

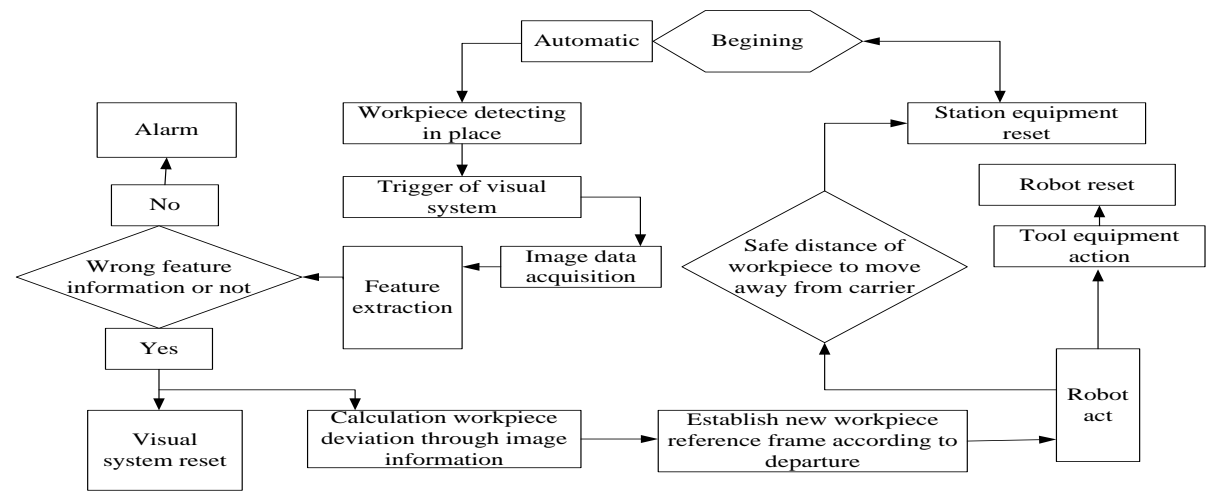

Figure 1. The work flow of vision-guided system

\section{The KEY Technologies of Robot Vision-Guided}

Dynamic grasping target work piece is a focus in the study of this article because many target work piece are movement together with the conveyor belt. Here the ready-made work and the main technique are briefly analyzed[6].

Image Segmentation. More classic methods including Threshold Segmentation and Clustering Segmentation. In recent years, the use of color image of the visual system is also increasing, many of the gray image segmentation method was used for color image division has also achieved good results.Based on global threshold segmentation method need to determine a threshold in advance, and then use the threshold for segmenting images, this method has some advantage,such as operation speed and simple algorithm. Suitable for the large difference of target and the background image, the weakness is threshold will not be able to change once established.The locally adaptive threshold image segmentation method is to use the method of change local threshold value. Select several threshold and used different thresholds for different regional division,so that make the threshold can adapt to the changes in local region.it could reduce some problem about the use of global threshold method for segmenting images between background and image which small differences of background. Dynamic method to determine the threshold depends on the pixel coordinates and the pixel gray value,dynamically determine threshold method has a large time complexity and space complexity,and this method is suitable for processing complex image detail and has good anti-interference.

The Method of Workpiece Image Segmentation based on Histogram Statistics. In this paper,in order to determine the threshold segmentation, the method based on the gray-level histogram statistics and analysis is used[7]. Because it is a gray image and the gray value range 0 from 255 , by using threshold segmentation, named $\mathrm{T}(0<\mathrm{T}<255)$ to cut the pixels in the image divided into two parts according to the grey value.The first part in the images of the corresponding background region, the gray value range 0 from $T$, the second part is the workpiece in the image, the gray value range $\mathrm{T}$ from 255. Suppose before image segmentation, the workpiece image is $f(x, y)$, after dealing with the threshold segmentation of image workpiece is $g(x, y)$, then:

$$
g(x, y)= \begin{cases}1 & f(x, y)>T \\ 0 & f(x, y)<T\end{cases}
$$

$T$ is the threshold of segmentation,the above formula can be used to split workpiece images. After the workpiece image is segmented and binaried,we can find that although there exist some miscellaneous points in images, it has already reached the the purpose of separate workpiece from 
the background image. Assume that an object's surface light that reflection can passes through the photocenter and projected onto imaging planes, in order to obtain the mathematical transformation relationship between the image and object space coordinates, the computer vision usually use the World Coordinate System $\left(x_{w}, y_{w}, z_{w}\right)$,Camera Coordinate System $\left(x_{c}, y_{c}, z_{c}\right)$ and Image Coordinate $\operatorname{System}\left(x_{u}, y_{u}\right)$.

World coordinate system is space coordinate of the target objects, use the coordinate system that reference points are free to choose to describe the position of the camera and the target object. Usually, the camera coordinate origin is photocenter, ${ }^{x_{c}}$ and ${ }^{y_{c}}$ respectively parallel to the imaging plane $^{x_{u}}$ and $y_{u}$, and $z_{c}$ perpendicular to the imaging plane and the principal point for camera is the intersection between $z_{c}$ and imaging planes. Image coordinate system is the description of three dimensional space information by projection transformation of $2 \mathrm{~d}$ plane images, description of the image coordinate system is often use two ways that in pixels and physical length.

Dynamic Grasping. The dynamic grasping of industrial robot vision-guided achieve the robot quickly grab of scattered the workpiece on the conveyor belt.In this paper,using the scheme of conveyor belt encoder feedback based on the robot,camera and conveyor are built to the dynamic grasping of industrial robot vision-guided system.Dynamic representation of a single target.Industrial intelligent camera fixed upper installed on the conveyor belt, in a certain interval to collect images, every collection of image through image processing to obtain the coordinates of the target artifact within sight and gathering image moment.It can be obtained at any time of the workpiece's current location coordinates through the the number of feedback pulses that installation on the conveyor belt speed encoder.The target location information can be expressed as $\left(x_{n}, y_{n}, N_{n}\right)$, at the process of conveyor belt running, at any time can get the current coordinates:

$$
(x, y)=\left(x_{n}+\left(N-N_{n}\right) K_{c}, y_{n}\right)
$$

above formula, $\mathrm{N}$ is the current time of the pulse value of conveyor belt speed encoder and the $N_{n}$ is the image acquisition time of in this processing, the $K_{c}$ is moving distance of the number of unit impulse

\section{Conclusion}

In recent years, industrial robotic vision-guided technology lays the foundation for application of the industrial robots in the smart line as a key technology of the practical robot research. In order to analyze the highly automated of industrial robot visual-guided system, a complete visual-guided system is introduced in this paper. Dynamic grasping target work piece is a focus on robot vision-guided,and the ready-made work and the main technique are also briefly analyzed in this paper.

\section{References}

[1] Sun D, Hu S, Shao X, et al. Global stability of a saturated nonlinear PID controller for robot manipulators[J]. IEEE Transactions on Control Systems Technology, 2009, 17(4): 892-899.

[2] Kang H J, Ro Y S. Robot manipulator modeling in Matlab-Sim Mechanics with PD control and online gravity compensation[C]. International Forum on Strategic Technology. October 13-15, 2010, Ulsan, Korea. 446-449.

[3] Su Y, Parra-Vega V. Global asymptotic saturated output feedback control of robot manipulators[C]. Proceedings of the 7 th World Congress on Intelligent Control and Automation. June 25-28, 2008, Chongqing, China. 3445-3450.

[4] Khoshelham K, Elberink S O. Accuracy and resolution of kinect depth data for indoor mapping applications[J]. Sensors, 2012, 12(2): 1437-1454. 
[5] Xiaofu Ma, Haris I. Volos, Xiangwei Zheng, Jeffrey H. Reed, and Tamal Bose. A Variation-Aware Approach for Task Allocation in Wireless Distributed Computing Systems[C], IEEE Global Communications Conference, 2013:5006-5011.

[6] Xia Yong,Feng Dagan,Wang Tianjiao.Image segmentation by clustering of spatial patterns.Pattern Recognition Letters, 2007, 28(12): 1548-1555.

[7] Du H, Henry P, Ren X, et al. Interactive 3D modeling of indoor environments with a consumer depth camera[C]//Proceedings of the 13th international conference on Ubiquitous computing. ACM, 2011: 75-84. 